\author{
What we do not know yet is whether PCTs will be \\ providing basic essential services from many \\ practices or just a few ....
}

\title{
OPINION
}

\section{Future changes in the GDS}

In the current series of interviews with bodies corporate in the $B D J$ there are a number of similar themes emerging that reflect the changes likely to happen within dentistry as a whole. One of these is the fascinating observation that it is the dentists in private practice who often complain the most about conditions in the NHS.

This phenomenon is not restricted to bodies corporate of course, but has simply emerged from the recent interviews. I can confirm that this is indeed a fairly common occurrence as I have myself occasionally witnessed emotive tirades against the NHS by dentists in (mainly) private practice. Apart from raising the obvious question 'Why are they so concerned when it doesn't affect them?' this emphasises the fact that for many years there has been a general discontent from the profession about the conditions in the NHS, especially in general practice. Interestingly this may be about to change in ways that many GDS dentists in England may be unaware of.

The reason I believe things are about to change was confirmed in the aftermath of the Queen's speech last month. In essence the forthcoming Bill to be presented to Parliament early next year is placing a duty on Primary Care Trusts (PCTs) to provide primary care dentistry to meet reasonable needs in their area, bringing dentistry into line with medical services. Thus the responsibility and the funding is moving from a central source to a local one, obliging PCTs to organise primary dental care as they see fit.

How will they meet this obligation? There are a number of options, including the PDS, Salaried Dental Services and contracts with individual practices, or any combination. We have already seen innovative arrangements between those in general practice, the community service and the hospital service in some of the PDS pilots. Doubtless we shall see more, with the possibility of GDPs in general practice receiving a salary directly from the PCT but working in a local dental practice.

What we do not know yet is whether PCTs will be providing basic essential services from many practices or just a few, whether extra (or enhanced) services will be provided from a few or none at all, what combination of payment methods will be used or how stable the funding arrangements will be from year to year.

What we do know is that funding will move from being non cash-limited (as it now is in the GDS where there is no specific limit on the total amount paid out to dentists each year depending on the claims submitted rather than a defined budget) to cashlimited. In other words the Government will be able to fix the total spend in advance, obviously preferable from their point of view. The good news is that the PCT will have to spend the funding allocation on dentistry rather than other areas of health, but exactly how it decides to do this will be up to each individual PCT and will doubtless vary from PCT to PCT, as local government funding already does in other areas such as the Business Links.

While much of this may seem somewhat distant from the day-to-day running of dental practices and the timetable is set for a year or two in the future, the ramifications of this switch are conceptually quite vast. For nearly 55 years general practice has meant that any qualified dentist can set up a general practice anywhere in the UK and expect funding from the NHS for all patients and treatment carried out under the GDS without major restrictions (other than prior approval for certain treatment items) on most dental treatment provided for their patients. That situation is about to change to one where localised control of the treatment funded will depend on the PCT meeting its obligations on the provision of 'reasonable' dental needs of the local population. We do not know how much of a change this new move will create in the working conditions of every practice yet, or if there will be much of a change at all. For some practices things will almost certainly improve, for others they may be worse.

What we can be sure of is that this is a major change in the way general dental care is provided, and as such will mean NHS dentistry will look very different in the future. No-one can say we do not live in interesting times.

Mike Grace m.grace@bda-dentistry.org.uk 